EGU2020-8316

https://doi.org/10.5194/egusphere-egu2020-8316

EGU General Assembly 2020

(c) Author(s) 2020. This work is distributed under

the Creative Commons Attribution 4.0 License.

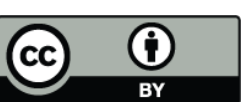

\title{
How to circumvent the limitations of open source software and orthorectify how (or better) than with commercial software
}

\author{
Valerio Baiocchi ${ }^{1}$, Roberta Onori ${ }^{2}$, Felicia Monti ${ }^{1}$, and Francesca Giannone ${ }^{3}$ \\ ${ }^{1}$ Sapienza University of Rome, DICEA, Italy (valerio.baiocchi@uniroma1.it) \\ ${ }^{2}$ SatCen, Madrid, Spain \\ ${ }^{3}$ Università Niccolò Cusano, Rome, Italy
}

High and very high resolution satellite images are now an irreplaceable resource for earth observation in general and for the extraction of hydrogeological information in particular. In order to use them correctly and compare them with previous surveys and maps, they must be treated geometrically to remove the distortions introduced by the acquisition process. Orthorectification is not a simple georeferencing because the process must take into account the three-dimensional acquisition geometry of the sensor. For this reason orthorectification must be performed within specific commercial software with additional costs compared to image acquisition which, in some cases, is currently free of charge.

Some orthorectification algorithms, mainly based on the RPC approach, are available in open source GIS software such as QGIS. OTB (Orpheus toolbox) for QGIS contains some of these algorithms but its interfaces are not clear and there are some incomprehensible limitations such as the impossibility to input three-dimensional ground control points (GCPs). This severely limits the final achievable accuracy because it does not allow to correctly estimate the influence of different ground morphologies on the acquisition geometry. To get around these limitations you can make a "pseudo DEM" and other expedients to complete the whole process obtaining absolute results comparable if not better than those of commercial software.

The proposed procedure may not be the fastest but it can be a valid alternative for those who use satellite images as a tool in their research work. 